\title{
Dependence of Electrical Field and Photoresponse on Multiplication Region Thickness for GaN APDs
}

\author{
Jingjing Wanyan, Zhaoqi Sun*, Shiwei Shi, Mingzai Wu, Gang He, Guang Li \\ School of Physics and Materials Science, Anhui University, Hefei 230039, China
}

\begin{abstract}
A visible-blind ultraviolet GaN back-illuminated avalanche photodiode with separate absorption and multiplication regions are simulated based on driftdiffusion equation. The current-voltage characteristics of the device have been numerically obtained. The result is in good agreement with the experimental data. It was found that the thickness of the multiplication layer is important to improve the electrical field profiles and spectral response characteristics. A peak responsivity of $106.5 \mathrm{~mA} / \mathrm{W}$ is achieved at $364 \mathrm{~nm}$ corresponding to the cutoff wavelength of GaN.
\end{abstract}

\section{I . INTRODUCTION}

High sensitivity ultraviolet avalanche photodetectors (APDs) have many important applications, including UV communications, biological detection and identification, and water quality monitoring [1]. The tremendous progress of $\mathrm{GaN} / \mathrm{AlGaN}$, GaN/AlN, and GaN/Si material growth technology in recent years makes these materials promising candidates for high-performance photodetectors operating in the UV spectral region [2] [3]. Currently, significant work has been done on the development of APDs using semiconductor materials with wide band gap such as $\mathrm{AlGaN}$ and GaN because the detectors can be inherently solar or visible blind, have low dark current, and operate in harsh environments [1, 4].

Ionization coefficients have been demonstrated to be higher for holes than that for electrons in $\mathrm{GaN}$ material [5]. The result makes back-illuminated separation of absorption and multiplication (SAM) GaN APDs well appropriate for reducing multiplication noise and enhancing gain through impact-ionization engineering [6]. Structure parameters of absorption and multiplication regions, playing critical roles in the performance of the device, have been widely investigated.

In this work, the current-voltage characteristics of $\mathrm{GaN}$ APDs with a SAM design allowing for nearly pure injection of holes into the multiplication region are numerically studied. Dependence of optical responsivity and electric field on the multiplication layer thickness is obtained.

\section{II . DEVICE STRUTURE AND SIMULATION MODELS}

\footnotetext{
* Corresponding author: zhaoqi_sun@yahoo.cn
}

For plain drift-diffusion simulation the well-known Poisson equation and continuity equations are used [7]. The carrier generation-recombination process consists of Shockley-Read-Hall (SRH), Radiative, Auger and optical generation-recombination terms. Additionally, the impact ionization model, tunneling effects (band-to-band tunneling) and avalanche multiplication model (van Overstraeten - de Man model) are included in the continuity equations [8].

The GaN SAM APD consists of a $p-i-n-i-n$ structure with the hole and electron concentrations of $(1-3) \times 10^{18}$ and (1$2) \times 10^{18} \mathrm{~cm}^{-3}$ for the p-type and n-type layers, respectively. The $i$ region is an undoped $\mathrm{GaN}$ with a residual carrier concentration of $(1-2) \times 10^{16} \mathrm{~cm}^{-3}$. Details of the structural information are shown in the inset of Fig.1.

\section{RESULTS AND DISCUSSIONS}

The calculated and measured current-voltage (I-V) characteristics are both shown in Fig. 1. The calculated $I-V$ curves are in good agreement with the experimental data [9], confirming the validity of our simulation model.

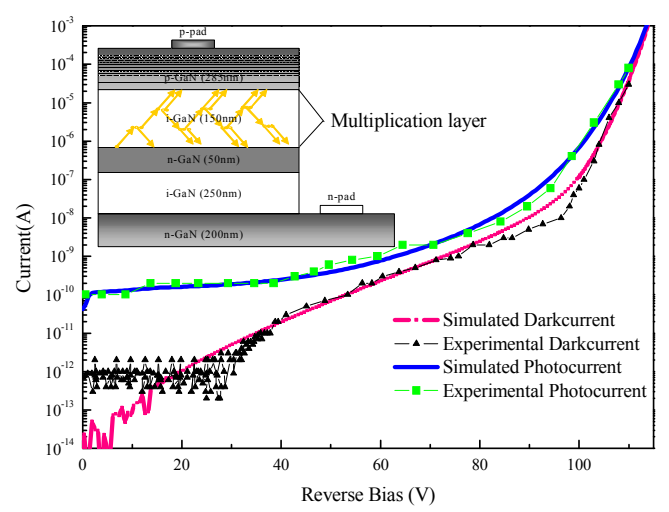

Figure1. The simulated and measured dark and light currents of the SAM APD. The inset shows the device structure.

The thickness of the multiplication region plays a critical role in the performance of the device. As the multiplication width is increased, the carriers can travel farther before reaching the opposite end of the junction and thus can contribute more multiplication events. However, an excessive increase of the multiplication width could possibly result in a 
decrease of device performance. The reason is that the ionization coefficients depend upon the electric field, in other words, the multiplication width. The thicker the multiplication region is, and the lower is the electric field strength for a given applied voltage, as shown in Fig. 2.

The inset of Fig. 2 shows a critical electric field of 2.8 $\mathrm{MV} / \mathrm{cm}$ [4] with the black line plotting the peak electric field at a reverse bias of $90 \mathrm{~V}$. The bias is well agreement recent experimental finding with the operating electric field of 80-100 $\mathrm{V}$ being optimal points for GaN avalanche APDs [10], for various thicknesses of the multiplication region. The result suggests that multiplication region should be designed between 100 and $150 \mathrm{~nm}$.

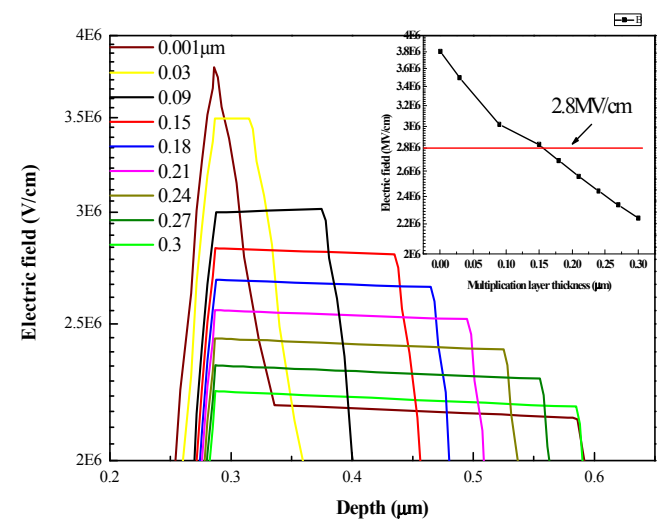

Figure 2. Electric field profiles of the SAM GaN diodes under $-90 \mathrm{~V}$. The inset shows the peak electric field as a function of the multiplication layer thickness.

The spectral response was measured under back illumination as a function of multiplication layer thickness under avalanche bias, as shown in Fig. 3. The optical power on the diode was $4.1 \mathrm{nW}$. The devices present a sharp response at around $364 \mathrm{~nm}$, corresponding to the absorption edge of GaN [9]. Responsivity increases when $\mathrm{W}_{\mathrm{m}}<\mathrm{W}_{\mathrm{p}}$ (the critical punchthrough thickness), because the increasing $\mathrm{W}_{\mathrm{m}}$ raises the depletion region thickness. When $\mathrm{W}_{\mathrm{m}}>\mathrm{W}_{\mathrm{p}}$, the device cannot be punched through, the response decreases. A peak responsivity of $106.5 \mathrm{~mA} / \mathrm{W}$ is achieved when $\mathrm{W}_{\mathrm{m}}$ is $0.10 \mu \mathrm{m}$.

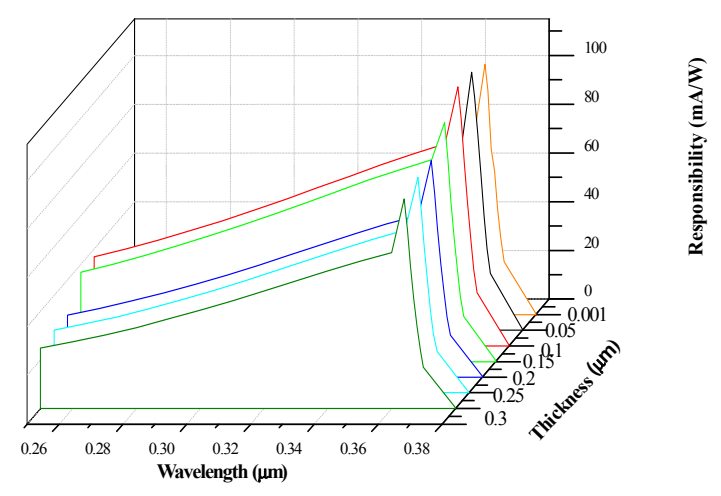

Figure .3 Variations of responsivities versus multiplication region thickness is calculated under avalanche bias.

\section{CONCLUSION}

GaN avalanche photodiodes with separated absorption and multiplication regions show great promise for realization of high gain detectors. Our results indicate that the APD can operate over a tunable spectrum in the near ultraviolet. The simulated $I-V$ curve is in good agreement with the experimental data. The dependence of electrical field profiles and spectral response on multiplication layer thickness has been discussed. A peak responsivity of $106.5 \mathrm{~mA} / \mathrm{W}$ is achieved at $364 \mathrm{~nm}$ corresponding to the cutoff wavelength of GaN.

\section{ACKNOWLEDGEMENTS}

The authors acknowledge the support provided by the State Key Program for Basic Research of China (2013CB632705), the National Natural Science Foundation of China (61290301).

\section{REFERENCES}

[1] M. Liu, X. Bai, C. Hu, X. Guo, J. C. Campbell, Z. Pan, M. M. Tashima, "Low dark count rate and high single-photon detection efficiency avalanche photodiode in Geiger-mode operation," IEEE Phot. Tech. Lett., 19, 378-80 (2007).

[2] X. D. Wang, W. D. Hu, X. S. Chen, J. T. Xu, X. Y. Li, W. Lu, "Photoresponse study of visible blind GaN/AlGaN p-i-n ultraviolet photodetector", Optical and Quantum Electronics, 42, Page 755-764 (2011).

[3].W. Hu, X. Chen, Z. Quan, C. Xia, W. Lu, "Demonstration and dynamic analysis of trapping of hot electron at gate edges model for current collapse and gate lag in GaN-based high-electron-mobility-transistor including selfheating effect", Applied Physics Letters, 89, 243501 (2006).

[4] R. McClintock, J. L. Pau, K. Minder, C. Bayram, P. Kung, and M. Razeghi, "Hole-initiated multiplication in back-illuminated $\mathrm{GaN}$ avalanche photodiodes,” Appl. Phys. Lett., 90, 141112 (2007).

[5] I. J. Oguzman, E. Bellotti, K. Brennan, J. Kolnik, R. Wang, and P. Ruden, "Theory of hole initiated impact ionization in bulk zincblende and wurtzite GaN", J. Appl. Phys., 81, 7827 (1997).

[6] X. Guo, L. B. Rowland, G. T. Dunne, J. A. Fronheiser, P. M. Sandvik, A.L. Beck, and J. C. Campbell, "Demonstration of ultraviolet separate absorption and multiplication $4 \mathrm{H}-\mathrm{SiC}$ avalanche photodiodes", IEEE Photonics Technol. Lett., 18, 136 (2006).

[7] $\mathrm{Hu}$ W D, Chen X S, Quan Z J, Xia C S, Lu W and Yuan H J, Demonstration and dynamic analysis of trapping of hot electrons at gate edge model for current collapse and gate lag in GaN-based high-electron-mobility transistor including self-heating effect, Appl. Phys. Lett., 89, 243501 (2006).

[8] X. Wang, W. Hu, X. Chen, J. Xu, L. Wang, X. Li, and W. Lu, Dependence of dark current and photoresponse characteristics on polarization charge density for GaN-based avalanche photodiodes, Journal of Physics D: Applied Physics, 44, 405102 (2011).

[9] J. L. Pau, C. Bayram, R. McClintock, M. Razeghi, and D. Silversmith, "Back-illuminated separate absorption and multiplication GaN avalanche Photodiodes", Appl. Phys. Lett., 92, 101120 (2008).

[10] Ryan McClintock, Erdem Cicek, Zahra Vashaei, Can Bayram, Manijeh Razeghi,nd Melville P. Ulmer, "III-Nitride Based Avalanche Photo Detectors", Proc. of SPIE, 7780 77801B-23501, 2012. 